\title{
Glutathione amperometric detection based on a thiol-disulfide exchange reaction
}

\author{
Francesco Ricci ${ }^{\mathrm{a}, *}$, Fabiana Arduini ${ }^{\mathrm{a}}$, Catalin S. Tuta ${ }^{\mathrm{a}}, \mathrm{Ugo}$ Sozzo ${ }^{\mathrm{a}}$, \\ Danila Moscone $^{\mathrm{a}}$, Aziz Amine ${ }^{\mathrm{b}}$, Giuseppe Palleschi ${ }^{\mathrm{a}}$ \\ a Dipartimento di Scienze e Tecnologie Chimiche, Università di Roma Tor Vergata, \\ Via della Ricerca Scientifica, 00133 Rome, Italy \\ ${ }^{\mathrm{b}}$ Faculté de Sciences et Techniques de Mohammadia, B.P. 146, Mohammadia, Morocco
}

Received 23 August 2005; received in revised form 17 October 2005; accepted 2 November 2005

Available online 20 December 2005

\begin{abstract}
A method based on a thiol-disulfide exchange reaction is proposed for glutathione detection. The method utilises a reaction between glutathione and an excess of the disulfide cystamine which produces an equimolar concentration of the thiol cysteamine. This latter is then detected at Prussian Blue modified screen printed electrodes at an applied potential of $200 \mathrm{mV}$ versus $\mathrm{Ag} / \mathrm{AgCl}$. First the cysteamine analytical parameters were optimised, resulting in a detection limit of $10^{-6} \mathrm{~mol}^{-1}$ and a linear range up to $10^{-4} \mathrm{~mol}^{-1}$. Reproducibility (R.S.D. $\left.=7 \%, n=6\right)$ and stability (more than 30 measurements with the same electrode) were satisfactory. Then the reaction between the disulfide cystamine and the thiol glutathione was optimised and a $\mathrm{pH}$ of 7.4 with a concentration of cystamine of $10^{-2} \mathrm{moll}^{-1}$ was chosen as the best conditions in terms of reaction rate and sensor sensitivity. Glutathione was then measured under the optimised conditions giving a detection limit of $2 \times 10^{-6}$ mol $1^{-1}$ and a linear range up to $5 \times 10^{-4} \mathrm{moll}^{-1}$. Blood samples were also tested in order to determine the recovery of the method. Recoveries between 92 and $103 \%$ were observed for glutathione concentrations in blood ranging from 0.5 to $3 \times 10^{-3} \mathrm{moll}^{-1}$.
\end{abstract}

(C) 2005 Elsevier B.V. All rights reserved.

Keywords: Glutathione; Screen printed electrode; Amperometric; thiol-disulfide exchange reaction

\section{Introduction}

Thiols are a class of compounds characterised by the presence of sulfhydryl residues [1]. The biological thiols can by classified as high-molecular mass protein thiols and low-molecular mass free thiols. Low-molecular weight thiols and disulfides, including glutathione (GSH), cysteine (Cys), and homocysteine (HCys), have numerous roles in metabolism and homeostasis [2].

Glutathione is a key physiological antioxidant that not only detoxifies reactive oxygen species directly, but also enhances the functional ability of other crucial antioxidants, such as Vitamins $\mathrm{E}$ and $\mathrm{C}[3,4]$. Altered levels of glutathione in plasma have been implicated in a number of pathological conditions, including Alzheimer's, Parkinson's diseases, diabetes, macular degeneration, and HIV disease [2].

\footnotetext{
* Corresponding author.

E-mail address: Francesco.ricci@uniroma2.it (F. Ricci).
}

Because of the importance and wide spread presence of glutathione and the possible use of its plasma levels as a biomarker of health status in many essential biological functions, its measurement is considered a primary goal in clinical chemistry [5].

However, glutathione measurement has always proven to be difficult. Most of the methods are time consuming, and either need derivatization or lack sufficient sensitivity [5].

A particularly prevalent chemical reaction of thiols is thiol-disulfide exchange in which the thiolate $\left(\mathrm{RS}^{-}\right)$acts as a nucleophile, displacing one sulfur atom in an existing disulfide bond and forming a new bond with the other sulfur atom [6].

One of the most common methods for thiols detection is the Ellman's test [5,7], which provides an accurate quantitative spectrophotometric measurement of thiols. It is based on the use of the thiol-disulfide exchange reaction between a thiol (RSH) and a disulfide $\left(\mathrm{R}^{\prime} \mathrm{SSR}^{\prime}\right)$ (Eq. (1)):

$$
\mathrm{RSH}+\mathrm{R}^{\prime} \mathrm{SSR}^{\prime} \rightleftarrows \mathrm{R}^{\prime} \mathrm{SSR}+\mathrm{R}^{\prime} \mathrm{SH}
$$


In the Ellman's test, the reactive disulphide is DTNB $\left(5,5^{\prime}\right.$ dithiobis-(2-nitrobenzoic acid)) and the sulphide produced in the reaction (Eq. (1)) is TNB having a yellow colour with a maximum absorption at $412 \mathrm{~nm}\left(\varepsilon=13,600 \mathrm{moll}^{-1} \mathrm{~cm}^{-1}\right)$ (Eq. (2)):

$$
\mathrm{RSH}+\mathrm{DTNB} \rightleftarrows \mathrm{R}^{\prime} \mathrm{SSR}+\mathrm{TNB}
$$

In the presence of an excess of DTNB the reaction is very fast and a quantitative measure of thiol can be achieved. This test has found a wide range of applications but has as major drawback, the fact that with coloured samples (such as blood) a sample pre-treatment is needed.

Compared to spectrophotometric methods, electroanalysis has the advantages of simplicity and high sensitivity and also the possibility of being used with coloured samples without any pre-treatment. However, the direct oxidation of thiols at conventional electrodes is slow and requires a potential of at least $1.0 \mathrm{~V}$ with problems of electrode passivation. For this reason, the use of chemically modified electrodes has been proposed for amperometric detection of thiols at low applied potentials $[8,9]$. In this perspective, the electrochemical determination of sulfhydryl compounds with cobalt phtalocyanine has been extensively studied [10,11]. Recently, the use of a modified screen printed electrode has been demonstrated to allow the detection of some important thiol at low applied potential (200 $\mathrm{mV}$ versus $\mathrm{Ag} / \mathrm{AgCl}$ ) due to the presence of Prussian Blue (PB) as mediator [12]. High amperometric signals with corresponding low detection limits were obtained for thiocholine and cysteamine. No signal, however, was observed for glutathione and other clinical important thiols such as homocysteine.

In this paper an application which relies on the high sensitivity towards cysteamine of the Prussian Blue modified electrodes is presented. The method proposed is based on a thiol-disulfide exchange reaction which is similar to the one used for the Ellman's test. However, the disulfide used as reagent is cystamine. A reduced thiol, in the presence of a high excess of this reagent, will react via a thiol-disulfide exchange reaction giving an equimolar concentration of cysteamine and a mixed disulfide (Eq. (3)):

$\mathrm{RSH}+$ cystamine $\rightarrow$ mixeddisulfide + cysteamine

The cysteamine produced is then detected with the Prussian Blue modified electrode. This electrochemical method has been optimised for use in the amperometric detection of glutathione in blood.

\section{Experimental}

\subsection{Apparatus}

Amperometric measurements were carried out using a VA 641 amperometric detector (Metrohm, Herisau, Switzerland), connected to a $X-t$ recorder (L250E, Linseis, Selb, Germany).

Cyclic voltammetry (CV) was performed using an Autolab/PGSTAT-12 electrochemical system (Eco Chemie, Utrecht, The Netherlands).

\subsection{Electrodes}

Screen-printed electrodes (SPEs) were produced with a 245 DEK (Weymouth, UK) screen printing machine. Graphite-based ink (Elettrodag 421) from Acheson (Milan, Italy) was used to print the working and counter electrode [21]. The substrate was a flexible polyester film (Autostat HT5) obtained from Autotype Italia (Milan, Italy). The electrodes were home produced in foils of 20. The diameter of the working electrode was $0.2 \mathrm{~cm}$ resulting in a geometric area of $0.031 \mathrm{~cm}^{2}$. Silver ink was used to print the reference electrode (see Ref. [21] for details of the electrode geometry). Before thiol measurement the reference electrode was clorurated by applying a potential of $0.6 \mathrm{~V}$ between the silver ink and an external $\mathrm{Ag} / \mathrm{AgCl}$ electrode for $20 \mathrm{~s}$ in a solution of $\mathrm{KCl} 0.1 \mathrm{moll}^{-1}$.

\subsection{Reagents}

All chemicals from commercial sources were of analytical grade.

All thiol solutions were prepared in $0.05 \mathrm{moll}^{-1}$ phosphate buffer $+0.1 \mathrm{~mol} \mathrm{l}^{-1} \mathrm{KCl}, \mathrm{pH} 5.5$. The standard solutions were made up daily in the same buffer. Before each measurement, the exact concentration of the thiol was evaluated using the spectrophotometric Elmann's test based on the reaction between thiol and DTNB (5, $5^{\prime}$-dithiobis-(2-nitrobenzoic acid)). For this purpose $900 \mu \mathrm{l}$ of a phosphate buffer solution $\left(0.05 \mathrm{moll}^{-1}\right), \mathrm{pH}$ 8.0 was mixed with $100 \mu \mathrm{l}$ of a DTNB solution $\left(10^{-3} \mathrm{moll}^{-1}\right)$. Then, the solution was spiked with $5 \times 10^{-5} \mathrm{~mol}^{-1}$ of thiol and the absorption was measured at $412 \mathrm{~nm}$.

For $\mathrm{pH}$ test, a Britton-Robinson universal (4-12 $\mathrm{pH}$ ) buffer $\left(0.05 \mathrm{moll}^{-1}\right.$ of phosphate, acetate and borate sodium salts $+\mathrm{KCl} 0.1 \mathrm{moll}^{-1}$ ) was used. $\mathrm{pH}$ was adjusted using $\mathrm{KOH}$ and $\mathrm{HCl}$.

\subsection{Preparation of PB modified screen printed electrodes}

Prior to PB modification, SPEs were pre-treated in a $0.05 \mathrm{moll}^{-1}$ phosphate buffer $+0.1 \mathrm{moll}^{-1} \mathrm{KCl}, \mathrm{pH} 7.4$ by applying a positive potential of $1.7 \mathrm{~V}$ for $3 \mathrm{~min}$. $\mathrm{PB}$ modification of SPEs was then accomplished by placing a drop $(10 \mu$ l total volume) of "precursor solution" onto the working electrode area. This solution is obtained by mixing $5 \mu \mathrm{l}$ of $0.1 \mathrm{moll}^{-1}$ potassium ferricyanide $\left(\mathrm{K}_{3} \mathrm{Fe}(\mathrm{CN})_{6}\right)$ in $10 \mathrm{mmol}^{-1} \mathrm{HCl}$ to $5 \mu \mathrm{l}$ of $0.1 \mathrm{~mol} \mathrm{l}^{-1}$ ferric chloride in $10 \mathrm{mmoll}^{-1} \mathrm{HCl}$. The drop was carefully pipetted exclusively on the working electrode area. The solution was left on the electrode for $10 \mathrm{~min}$ and then rinsed with a few millilitres of $10 \mathrm{mmol}^{-1} \mathrm{HCl}$. The electrodes were then left $90 \mathrm{~min}$ in the oven at $100{ }^{\circ} \mathrm{C}$ to obtain a more stable and active layer of Prussian Blue [15].

The PB modified electrodes were stored dry at room temperature in the dark.

\subsection{Cysteamine measurements}

Measurements of cysteamine were performed using amperometric batch analysis in a stirred phosphate buffer solution 
(phosphate $\left.0.05 \mathrm{moll}^{-1}+\mathrm{KCl} 0.1 \mathrm{moll}^{-1}, \mathrm{pH} 7.4\right)(5 \mathrm{ml})$ with an applied potential of $+200 \mathrm{mV}$ versus $\mathrm{Ag} / \mathrm{AgCl}$. When a stable baseline current was reached, the cysteamine was added and the response was recorded after $15 \mathrm{~s}$.

\subsection{Glutathione measurements using thiol-disulfide exchange reaction}

In the electrochemical procedure proposed in this paper for glutathione measurements, the sensors were used in amperometric batch mode. The PB electrode was immersed in the working buffer $\left(0.05 \mathrm{moll}^{-1}+\mathrm{KCl} 0.1 \mathrm{moll}^{-1}, \mathrm{pH} 7.4\right)$ containing a certain amount of cystamine $\left(10^{-2} \mathrm{moll}^{-1}\right)$. When a stable baseline current was obtained (applied potential $=200 \mathrm{mV}$ versus $\mathrm{Ag} / \mathrm{AgCl}$ ), the desired concentration of glutathione was added to the solution and after $30 \mathrm{~s}$ the signal was recorded.

\subsection{Glutathione measurements in blood samples}

For glutathione measurements in human blood samples, the blood samples were first centrifuged for $10 \mathrm{~min}$ at $6000 \mathrm{rpm}$ in order to separate erythrocytes from serum. After this, the erythrocytes were collected and haemolysed by diluting with distilled water $(1: 2)$.

The PB electrode was immersed in the working buffer containing the chosen amount of cystamine $\left(10^{-2} \mathrm{moll}^{-1}\right)$. When a stable baseline current was obtained (at an applied potential $=200 \mathrm{mV}$ versus $\mathrm{Ag} / \mathrm{AgCl}$ ), the haemolysed erythrocytes were added to the buffer $(50 \mu \mathrm{l}$ in $5 \mathrm{ml})$ and when a stable baseline was again reached, a concentration of glutathione $\left(10^{-5} \mathrm{~mol}^{-1}\right)$ was added to the solution and after $30 \mathrm{~s}$ the signal was recorded $\left(I_{0}\right)$. The electrode was then rinsed and a new measurement was performed. In this case the blood sample was spiked with glutathione $\left(10^{-3} \mathrm{~mol}^{-1}\right)$ and injected in the buffer $(50 \mu \mathrm{lin} 5 \mathrm{ml})$ to reach a concentration of $10^{-5} \mathrm{~mol} \mathrm{l}^{-1}$. The signal $\left(I_{1}\right)$, recorded after $30 \mathrm{~s}$, was used to calculate the recovery with the formula:

recovery $=\left(I_{1}-I_{0}\right) / I_{0} \times 100$

The same procedure was adopted with other blood samples spiked with different concentrations of glutathione $(0.5,2$ and $\left.3 \times 10^{-3} \mathrm{moll}^{-1}\right)$. For each concentration, sample and recovery measurements were made in triplicate.

\section{Results and discussion}

\subsection{Catalytic oxidation of cysteamine by Prussian Blue}

In a previous paper it was demonstrated that Prussian Blue (PB) modified electrodes have a strong catalytic activity towards thiocholine and cysteamine [12].

Cysteamine oxidation at the PB modified SPE has been demonstrated performing voltammetric experiments. Fig. 1 compares the cyclic voltammograms of PB modified electrodes in the presence and absence of cysteamine $\left(3 \times 10^{-3} \mathrm{moll}^{-1}\right)$. The increase of oxidation current observed in the presence of

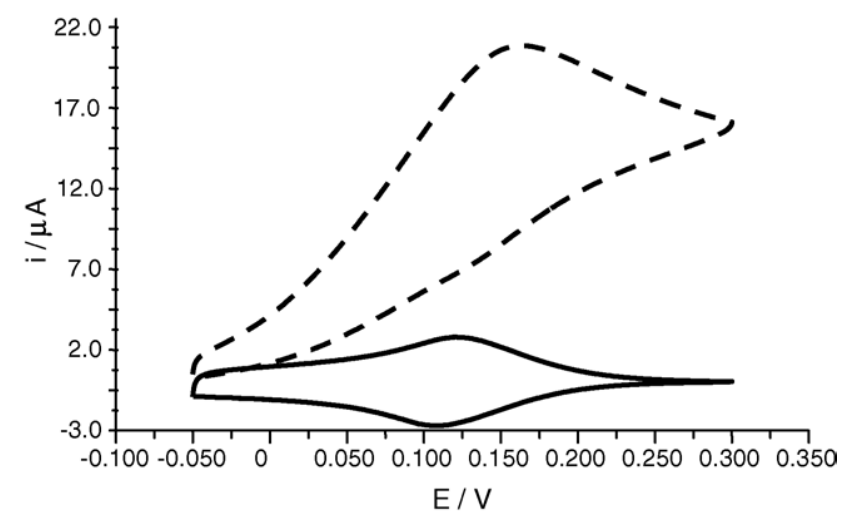

Fig. 1. Cyclic voltammograms showing the catalytic oxidation of cysteamine at a PB modified SPE. Cyclic voltammograms obtained with modified electrodes at a scan rate of $10 \mathrm{mV} \mathrm{s}^{-1}$. About $0.05 \mathrm{moll}^{-1}$ phosphate buffer $+0.1 \mathrm{moll}^{-1}$ $\mathrm{KCl}$, pH 7.4 - solid line Prussian Blue modified electrode in phosphate buffer; dotted line: Pussian Blue modified electrode in phosphate buffer in the presence of $3.0 \mathrm{mmol}^{-1}$ cysteamine

cystamine appears in correspondence to the formation of the oxidised form of $\mathrm{PB}\left(\mathrm{Fe}^{\mathrm{III}} \mathrm{Fe}^{\mathrm{II}}(\mathrm{CN})_{6}\right)$ suggesting a catalytic effect of such a mediator for the oxidation of cysteamine.

The reactions that occur on the electrode are as follows:

$\mathrm{PB}_{\mathrm{ox}}+$ cysteamine $(\mathrm{RSH}) \rightleftarrows \mathrm{PB}_{\text {red }}+$ cystamine $(\mathrm{RSSR})$,

$\mathrm{PB}_{\text {red }}+$ electrode $\rightleftarrows \mathrm{PB}_{\mathrm{ox}}+$ electrode

Despite the fact that the signal for cysteamine was large and a clear catalytic activity was observed at Prussian Blue modified electrodes, no signal was observed for glutathione. This could be related to both the high-molecular mass and to the presence of a negative charge on this compound. Both these parameters were in fact demonstrated to negatively affect the catalytic activity of PB [12]. Also in amperometric measurements no signal was obtained for high concentrations of glutathione at an applied potential of $200 \mathrm{mV}$.

The catalytic activity of PB towards cysteamine oxidation starts at around $100 \mathrm{mV}$ reaching a maximum at $150 \mathrm{mV}$; for this reason, $200 \mathrm{mV}$ was chosen as the potential to be applied for amperometric measurement of cysteamine.

\subsection{Cysteamine amperometric detection}

The cysteamine measurements were performed in amperometric batch mode at a $\mathrm{pH}$ of 7.4 which had previously been demonstrated to be suitable for thiol detection with PB modified electrodes [12].

The Prussian Blue modified electrodes were able to detect cysteamine at concentrations as low as $10^{-6} \mathrm{moll}^{-1}(S / N=3)$. This detection limit is slightly higher than that obtained with cobalt phtalocyanine modified electrodes which at present are the most used probes for amperometric thiol detection [10]. However, the PB modified electrodes seem to represent an improvement due to their very high stability which allows numerous successive measurements using the same electrode without loss of sensitivity. The signal was linear up to a concentration of $10^{-4} \mathrm{~mol}^{-1}$ and a sensitivity of $0.37 \mathrm{~mA} / \mathrm{M} \mathrm{cm}^{2}$ was 
Table 1

Analytical parameters of Prussian Blue modified screen printed electrodes for cysteamine and glutathione

\begin{tabular}{|c|c|c|c|c|c|c|c|}
\hline Analyte & $\begin{array}{l}\text { Detection limit } \\
\left(\mu \mathrm{moll}^{-1}\right)\end{array}$ & $\begin{array}{l}\text { Noise } \\
\left(\mathrm{mA} \mathrm{cm}^{-2}\right)\end{array}$ & $\begin{array}{l}\text { Linearity range } \\
\left(\mathrm{mmol}^{-1}\right)\end{array}$ & $\begin{array}{l}\text { Sensitivity } \\
\left(\mathrm{mA} / \mathrm{M} \mathrm{cm}^{2}\right)\end{array}$ & $\begin{array}{l}\text { R.S.D. }(\%), \\
n=6\end{array}$ & $\begin{array}{l}\text { Time to reach stable } \\
\text { background (s) }\end{array}$ & $\begin{array}{l}\text { Time to reach } 90 \% \\
\text { of the signal (s) }\end{array}$ \\
\hline Cysteamine & 1.0 & 0.2 & $1.0 \times 10^{-3}-0.1$ & 0.37 & 7 & 20 & 5 \\
\hline Glutathione & 2.0 & 0.2 & $2.0 \times 10^{-3}-0.5$ & 0.26 & 7 & 20 & 15 \\
\hline
\end{tabular}

Batch amperometric analysis, applied potential $=200 \mathrm{mV}$ vs. $\mathrm{Ag} / \mathrm{AgCl}$. Phosphate buffer $0.05 \mathrm{~mol}^{-1}+\mathrm{KCl} 0.1 \mathrm{~mol} 1^{-1}$, $\mathrm{pH} 7.4$. In the case of glutathione the phosphate buffer was added with $10^{-2} \mathrm{moll}^{-1}$ of cystamine.

obtained (see Table 1). The reproducibility of the PB modified screen printed electrodes was also calculated and a R.S.D. of $7 \%$, obtained with six different sensors, was observed. After the injection of cysteamine, the signal reached a stable current after $10 \mathrm{~s}$ while $5 \mathrm{~s}$ are needed to obtain $90 \%$ of the final signal.

Even if screen printed electrodes are usually adopted as disposable sensors due to their low cost and the possibility of massproduction, these sensors can also be used with no problems and no loss of PB activity for more than 30 single measurements of cysteamine since no passivation effect is observed due to this thiol.

The sensitive detection of cysteamine at PB modified electrodes, was then exploited for the amperometric detection of glutathione in blood samples via a thiol-disulfide exchange reaction. A similar approach was proposed by Compton's group in 2002 [13] using a two-step reaction in which DTNB and DEPD ( $N, N$-diethyl- $p$-phenylenediamine) were used as reagents, with linear sweep voltammetry detection. Problems due to high concentration of interferents such as ascorbic acid or uric acid could, however, occur with this approach.

\subsection{Glutathione amperometric detection with a thiol-disulfide exchange reaction}

The method, already described in Section 1, is based on a thiol-disulfide exchange reaction (similar to that used on the Ellman's test) where the final product would be cysteamine. Given that the disulfide cystamine (the oxidised form of cysteamine, RSSR) is the main reagent, several parameters have been investigated and optimised such as the concentration of cystamine, $\mathrm{pH}$, time of the reaction, and also the working medium. Regarding glutathione detection, some relevant information should be considered. Glutathione is a tripeptide thiol which has its highest concentration in erythrocytes $\left((1-3) \times 10^{-3} \mathrm{moll}^{-1}\right)$. The intracellular level of glutathione is much greater than that of cysteine, cystine and other thiols, and represents almost $99 \%$ of total thiols [5]. Glutathione is normally degraded by reaction with oxygen and the rapid bonding with protein residues. For this reason the analysis time should be kept as low as possible. To achieve this, the cystamine concentration and the $\mathrm{pH}$ buffer have to be thoroughly investigated in order to make the reaction fast and complete in a short time.

\subsubsection{Study of the thiol-disulfide exchange reaction}

The reaction between glutathione and cystamine was initially studied using a fixed concentration of glutathione $\left(10^{-5} \mathrm{moll}^{-1}\right)$ and varying the concentration of cystamine in the working buffer. As can be seen in Fig. 2, the rate of the reaction increases with cystamine concentration up to a limiting value of $10^{-2} \mathrm{moll}^{-1}$. This experiment was performed also with higher concentration of glutathione (up to $10^{-4} \mathrm{moll}^{-1}$ ) giving similar results thus demonstrating that a cystamine concentration of $10^{-2} \mathrm{~mol} \mathrm{l}^{-1}$ is sufficient to obtain a rapid and equimolar response to different amounts of glutathione in $15 \mathrm{~s}$. This time can also be considered low enough to avoid any degradation of the glutathione during the measurement.

In addition, the optimum $\mathrm{pH}$ for the reaction between glutathione and cystamine has been carefully studied. In the case of thiol-disulfide exchange reaction, a basic $\mathrm{pH}$ is always required to obtain a fast and complete reaction. This is due to the fact that at basic $\mathrm{pH}$ most thiols are in the thiolate form $\left(\mathrm{RS}^{-}\right)$which is well known to be more reactive than the protonated one [14]. A plot of the $\mathrm{pH}$ dependence of the reaction rate is shown in Fig. 3. At more acidic conditions the reaction rate is very slow and at $\mathrm{pH} 6$ the signal obtained at $30 \mathrm{~s}$ is only $9 \mathrm{nA}$. At this $\mathrm{pH}, 60 \mathrm{~s}$ are needed to obtain a stable signal. At pH 7.4 a stable signal is obtained after only $15 \mathrm{~s}$ and a current of about $80 \mathrm{nA}$ is recorded. At pH 8.0 and 9.0 the time needed to reach a stable signal is the same as that recorded at $\mathrm{pH} 7.4$, due to the fact that $15 \mathrm{~s}$ are the minimum time for stabilisation of cysteamine signal at the PB modified electrode. Using higher concentrations of glutathione the same time for current stabilisation was observed. Thus for

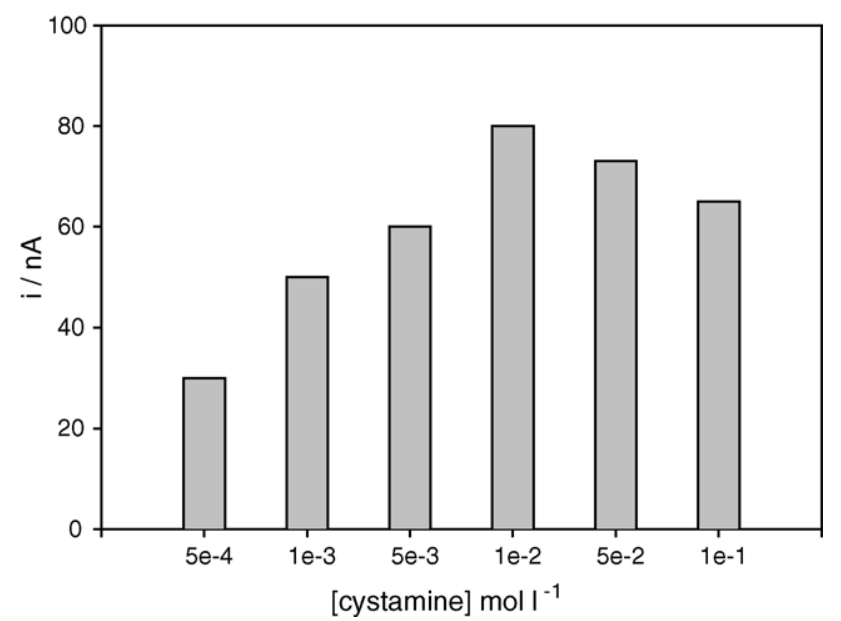

Fig. 2. Influence of cystamine concentration in the thiol-disulfide exchange reaction between cystamine and glutathione. In the $y$-axis are shown the current signal due to the formation of cysteamine and recorded after $30 \mathrm{~s}$ from the injection of $10^{-5} \mathrm{moll}^{-1}$ glutathione. Measurements carried out at $200 \mathrm{mV}$ vs. $\mathrm{Ag} / \mathrm{AgCl}$ in phosphate buffer $0.05 \mathrm{moll}^{-1}+\mathrm{KCl} 0.1 \mathrm{moll}^{-1}$, pH 7.4. 


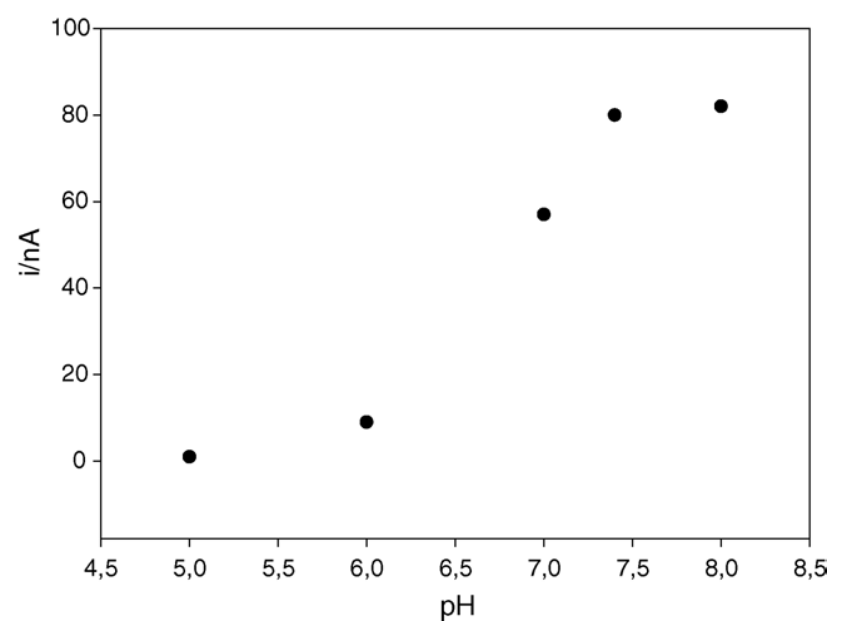

Fig. 3. Influence of $\mathrm{pH}$ in the thiol-disulfide exchange reaction between cystamine and glutathione $\left(10^{-5} \mathrm{moll}^{-1}\right)$. The current signal obtained after $30 \mathrm{~s}$ and due to the formation of cysteamine is shown in the $y$-axis. Measurements carried out at $200 \mathrm{mV}$ vs. $\mathrm{Ag} / \mathrm{AgCl}$ in phosphate buffer $0.05 \mathrm{moll}^{-1}+\mathrm{KCl} 0.1 \mathrm{moll}^{-1}$, $\mathrm{pH} 7.4,10^{-2} \mathrm{moll}^{-1}$ cystamine.

further studies, a pH of 7.4 was chosen which is low enough to avoid glutathione degradation during measurements. Moreover, this $\mathrm{pH}$ is suitable for use with PB modified electrodes [20].

\subsubsection{Measurement of glutathione standard solutions}

After the optimisation of the analytical parameters, glutathione standard solutions were run. The procedure is described in Section 2 and is summarised in Fig. 4 where an original recording for glutathione standard additions is depicted. Under the optimised conditions, the PB modified screen printed electrodes were able to detect glutathione at a concentration of $2 \times 10^{-6} \mathrm{moll}^{-1}$ with a linearity up to $5 \times 10^{-4} \mathrm{moll}^{-1}$. The sensitivity in this case was $0.26 \mathrm{~mA} / \mathrm{M} \mathrm{cm}^{2}$, which is somewhat lower than that obtained for cysteamine (see Table 1). This is due to the presence of an excess of cystamine in the working buffer which slightly decreases the sensitivity of the PB modified electrodes. However, the detection limit and linear range are similar to those obtained by Compton group with a similar approach [13] and are still good enough to allow a measurement of glutathione in real samples with a sufficient dilution factor. The same reproducibility obtained previously with cysteamine (R.S.D. $=7 \%, n=6$ ) was also confirmed. The operational stability of the sensor was the same observed for cysteamine and was not affected by the presence of glutathione.

\subsection{Interference study}

Some possible electrochemical interferences such as acetaminophen, ascorbic acid, and uric acid as well as those represented by biological thiols such as cysteine and homocysteine (chemical interferences which could react with the reagent (cystamine) giving cysteamine) have also been tested. In Table 2 are summarised the signals obtained with these compounds, compared with the current value of glutathione. In the case of the electrochemical interferences, no response was observed for uric acid and acetaminophen, while a signal which represents ca. $7 \%$

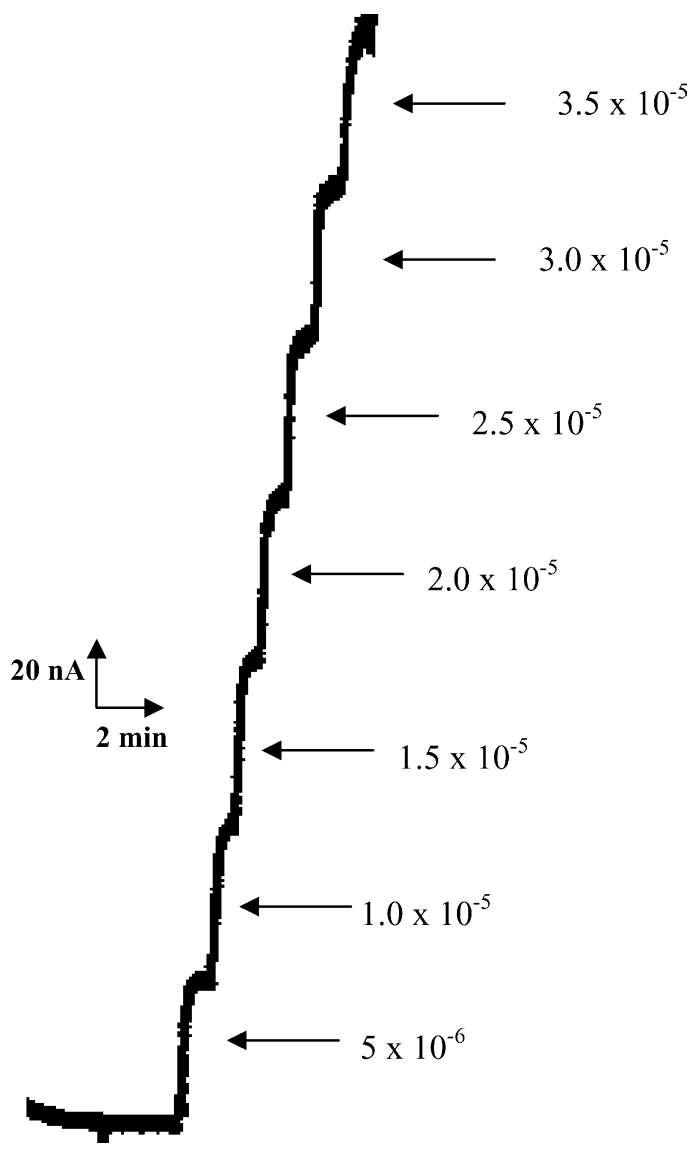

Fig. 4. Original recordings obtained with the method proposed for glutathione detection. PB modified SPEs were used in batch amperometric mode $(200 \mathrm{mV}$ vs. $\mathrm{Ag} / \mathrm{AgCl})$ in stirred solution. Standard additions of $5 \mu \mathrm{l}$ of a solution $10^{-2} \mathrm{moll}^{-1}$ of glutathione were added in a $10 \mathrm{ml}$ phosphate buffer $0.05 \mathrm{moll}^{-1}+\mathrm{KCl} 0.1 \mathrm{moll}^{-1}, \mathrm{pH} 7.4+10^{-2} \mathrm{moll}^{-1}$ of cystamine to get a final concentration of $5 \times 10^{-6}, 10^{-5}, 1.5 \times 10^{-5}, 2.0 \times 10^{-5}, 2.5 \times 10^{-5}$, $3.0 \times 10^{-5}, 3.5 \times 10^{-5} \mathrm{moll}^{-1}$ of glutathione.

of the glutathione signal is obtained with ascorbic acid. For the chemical interferences, as expected given that the thiol-disulfide exchange reaction functions for almost all the thiols, the same signal was obtained for cysteine and homocysteine. It is important to stress at this point, that the method proposed is not a selective method for glutathione, but represents a method for the determination of total thiol content. Being a common reaction for all the thiols, it could be used for different purposes, ranging from the detection of thiols in pharmaceutical products to the determination of thiols in biological fluids. However, it should be noted that in erythrocytes the concentration of thiols other than glutathione would represent only $1 \%$ of the glutathione content. This is also the case for ascorbic acid, whose concentration in the blood is around $25-100 \mu \mathrm{moll}^{-1}$ with a maximum value of $200 \mu \mathrm{mol}^{-1}$. This means that the concentration of ascorbic acid is almost 10 times lower than the average glutathione concentration and thus its overall interference effect will be almost negligible.

All these interferents compounds were also studied to understand if the glutathione detection could be affected by their presence. The glutathione signal was the same in the presence of all the compounds tested (each at a concentration of $10^{-5} \mathrm{moll}^{-1}$ ) 
Table 2

Response of the Prussian Blue modified screen printed electrode towards interfering species

\begin{tabular}{|c|c|c|c|c|}
\hline $\begin{array}{l}\text { Class of } \\
\text { interferences }\end{array}$ & Compound & $\begin{array}{l}\text { Concentration tested } \\
\left(\mathrm{moll}^{-1}\right)\end{array}$ & $\begin{array}{l}\text { Current signal } \\
\text { given }(\mathrm{nA})\end{array}$ & $\begin{array}{l}\text { Percentage of } \\
\text { signal }\end{array}$ \\
\hline & Glutathione & $10^{-5}$ & 80 & 100 \\
\hline \multirow[t]{3}{*}{ Electrochemical } & Uric acid & $5 \times 10^{-5}$ & - & 0 \\
\hline & Acetaminophen & $5 \times 10^{-5}$ & - & 0 \\
\hline & Ascorbic acid & $10^{-5}$ & 6 & 7.5 \\
\hline \multirow[t]{2}{*}{ Chemical } & Cysteine & $10^{-5}$ & 81 & 101 \\
\hline & Homocysteine & $10^{-5}$ & 80 & 100 \\
\hline
\end{tabular}

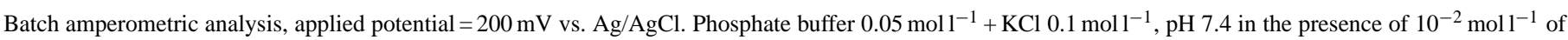
cystamine.

and no effect was observed in the overall sensitivity of the PB modified electrodes.

Another important point for the application of this method in real blood samples regards the presence of additives such as EDTA and citrate that are usually introduced soon after the blood sampling as anticoagulants [16-18]. In the presence of EDTA, the PB modified electrode showed a lower sensitivity towards glutathione and a constant decrease over time in the current signal. The same behaviour was observed when only cysteamine (in presence of EDTA) was used as analyte demonstrating that EDTA does not affect the thiol-disulfide exchange reaction. The presence of EDTA seems to cause the degradation of Prussian Blue and an inactivation of its catalytic activity. This hypothesis seems to be confirmed by a cyclic voltammetry study in which the Prussian Blue modified electrode was tested before and after 30 min of continuous work in a buffer containing EDTA. A consistent decrease in the oxidation and reduction peaks of Prussian Blue was observed (data not shown). As a control, cyclic voltammetry was also performed after $30 \mathrm{~min}$ of work in phosphate buffer without EDTA. No appreciable differences could be observed when compared with the first cycle. The mechanism of the effect of EDTA is still not clear. It is known that EDTA is a strong complexing agent for many metal ions such as $\mathrm{Ca}, \mathrm{Fe}$, etc. and it is possible that the presence of EDTA in the working buffer leads to a complexation of the external iron atoms which constitute the backbone of the Prussian Blue [20]. This could cause two different effects: one related to the leakage of the deposited Prussian Blue through disruption of its structure, the other a sort of passivation of the electrode surface with a subsequent decrease of the catalytic activity and of the current signal. Both these effects will lead to a decrease of the oxidation and reduction peaks as observed in cyclic voltammetry experiments. However, the negative effect of EDTA could be overcome by including the appropriate amount of divalent cations such as $\mathrm{Ca}^{2+}$ or $\mathrm{Mg}^{2+}$ in the working buffer. This has been confirmed by using a concentration of $10^{-2} \mathrm{moll}^{-1}$ of $\mathrm{CaCl}_{2}$ and in presence of $10^{-3} \mathrm{~mol}^{-1}$ of EDTA. In this case the stability of the Prussian Blue modified electrode was the same as that obtained in absence of EDTA and also the sensitivity towards cysteamine was not affected.

Another anticoagulant frequently used for clinical purposes is citrate. In this case, no effect was observed either in cyclic voltammetry experiment or in amperometric batch analysis. The analytical parameters observed for cysteamine and glutathione were in fact not altered by the presence in the working buffer of citrate and no decrease in the stability of the Prussian Blue layer was observed. The different behaviours of EDTA and citrate is probably related to their different binding affinities for $\mathrm{Fe}^{2+}$ [19]. The results lead to the conclusion that for glutathione measurement with Prussian Blue modified electrodes, blood samples treated with citrate pose no problem, while in the case of EDTA, a prior addition of $\mathrm{Ca}^{2+}$ can prevent interference.

\subsection{Measurement of glutathione in blood samples}

Finally, the method presented and optimised in this paper was tested with blood samples in order to understand its effective suitability for clinical applications.

Table 3

Blood samples measurements

\begin{tabular}{|c|c|c|c|c|}
\hline $\begin{array}{l}\text { Blood sample glutathione } \\
\text { concentration }^{\mathrm{a}}\left(\times 10^{-3} \mathrm{moll}^{-1}\right)\end{array}$ & $\begin{array}{l}\text { Glutathione concentration in the working } \\
\text { solution }^{\mathrm{b}}\left(\times 10^{-5} \mathrm{moll}^{-1}\right)\end{array}$ & Detected value & Recovery $^{c}$ & R.S.D. $(\%), n=3$ \\
\hline 0.5 & 0.50 & 0.49 & 98 & 4 \\
\hline 1.0 & 1.00 & 1.03 & 103 & 8 \\
\hline 2.0 & 2.00 & 1.90 & 95 & 8 \\
\hline 3.0 & 3.00 & 2.75 & 92 & 7 \\
\hline
\end{tabular}

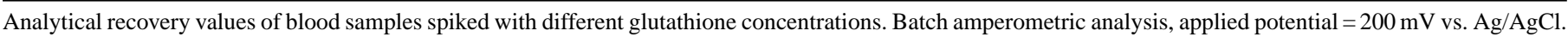

Phosphate buffer $0.05 \mathrm{moll}^{-1}+\mathrm{KCl} 0.1 \mathrm{moll}^{-1}, \mathrm{pH} 7.4+10^{-2}$ mol $1^{-1}$ of cystamine. See Section 2 for details on the procedure.

a Glutathione spikes in the blood sample.

b This value is obtained by dividing the spiked blood concentration by the dilution factor 100 (i.e. $50 \mu \mathrm{l}$ of blood in $5 \mathrm{ml}$ of buffer).

c Mean value obtained for three triplicate measurements. 
However, being the normal levels of other thiols and especially of cysteine in serum about $0.3 \times 10^{-3} \mathrm{~mol}^{-1}$, which represents a high percentage if compared with glutathione content in erythrocytes, a separation of the serum from the erythrocytes is needed to avoid any interference.

The final dilution of the erythrocytes was 100-fold, which also avoids the majority of electrochemical interferences. In order to demonstrate this, the samples were first tested in a buffer solution in the absence of cystamine. In this case we did not observe any appreciable signal after the addition of the sample.

The blood samples were then spiked with different concentrations of glutathione and the recovery values were calculated. As summarised in Table 3 , for all the concentrations tested $(0.5$, $1,2,3 \times 10^{-3} \mathrm{moll}^{-1}$ ), good recovery values were obtained. The results demonstrate that the thiol-disulfide exchange reaction is not influenced by the presence of a complex matrix. Both the time and completion of the reaction are similar of those obtained in standard solutions. Glutathione was tested in the concentration range which could be found in normal and pathologic conditions, with no substantial differences in the recovery values.

\section{Conclusions}

An analytical method based on a thiol-disulfide exchange reaction for glutathione detection has been developed. The method allows glutathione detection via the reaction between an excess of the disulfide cystamine which produces an equimolar concentration of the thiol cysteamine. The cysteamine produced is then amperometrically detected at PB modified electrode at an applied potential of $200 \mathrm{mV}$ versus $\mathrm{Ag} / \mathrm{AgCl}$. First the cysteamine detection parameters were optimised $\left(\mathrm{LOD}=10^{-6} \mathrm{moll}^{-1}\right.$, linear range up to $10^{-4} \mathrm{moll}^{-1}$ ). After this the reaction between the disulfide cystamine and the thiol glutathione was optimised and glutathione was measured with the proposed method giving a detection limit of $2 \times 10^{-6} \mathrm{moll}^{-1}$ and a linear range up to $5 \times 10^{-4} \mathrm{moll}^{-1}$. Blood samples were also tested in order to determine the recovery of the method. Values of recovery between 92 and $103 \%$ were observed for glutathione concentrations in blood ranging from 0.5 and $3 \times 10^{-3} \mathrm{moll}^{-1}$. It was then demonstrated that the method has promise for applications to glutathione determination in complex matrix samples. Further studies are needed in order to optimise the method. Care must be taken to avoid glutathione degradation before the measurement as well as interferences from other thiols. Also, the possibility to measure the total glutathione content which would be the sum of reduced (GSH) and oxidised (GSSG) glutathione should be studied. This might be possible by introducing a quantity of glutathione reductase to convert all the oxidised glutathione into the reduced form. This is particularly important if one bears in mind that an important parameter regarding glutathione is the ratio between reduced and oxidised forms.

\section{References}

[1] R. Singh, G.M. Whitesides, in: S. Patai, Z. Rappoport (Eds.), The Chemistry of Sulphur-containing Functional Groups, John Wiley and Sons, Chichester, UK, 1993.

[2] W.A. Kleinman, J.P.R. Richie, Biochem. Pharmacol. 60 (2000) 19.

[3] A. Mister, M.E. Anderson, Annu. Rev. Biochem. 52 (1983) 711.

[4] W. Droge, C. Pottmeuer-Gerber, H. Schimdt, S. Nick, Immunology 172 (1986) 151.

[5] A. Pastore, G. Federici, E. Bertini, F. Piemonte, Clin. Chim. Acta 333 (2003) 19.

[6] G. Bulai, T. Kortemme, D.P. Goldenberg, Biochemistry 37 (1998) 8965

[7] F. Worek, U. Mast, D. Kiderlen, C. Diepold, P. Eyer, Clin. Chim. Acta 288 (1999) 73.

[8] B. Filanovsky, Anal. Chim. Acta 394 (1999) 91.

[9] P. Calvo-Marzal, K.Y. Chumbimuni-Torres, N.F. Hoer, G. de Oliveira Neto, L.T. Kubota, Sens. Actuators B 100 (2004) 337.

[10] J.P. Hart, I.C. Harley, Analyst 119 (1994) 259.

[11] A. Napier, J.P. Hart, Electroanalysis 8 (1996) 1006.

[12] F. Ricci, F. Arduini, A. Amine, D. Moscone, G. Palleschi, J. Electroanal. Chem. 563 (2004) 229.

[13] O. Nekrassova, P.C. White, S. Threlfell, G. Hignett, A.J. Wain, N.S Lawrence, J. Davis, R.G. Compton, Analyst 127 (2002) 797.

[14] M. Friedman, The Chemistry and Biochemistry of the Sulphydryl Group in Amino Acids, Peptides and Proteins, 1st ed., Pergamon Press, Oxford, 1973.

[15] F. Ricci, A. Amine, G. Palleschi, D. Moscone, Biosens. Bioelectr. 18 (2003) 165 .

[16] S. Caliskan, F. Kuralay, B. Onvural, Clin. Chim. Acta 309 (2001) 53.

[17] M.J. Evans, J.H. Livesey, M.J. Ellis, T.G. Yandle, Clin. Biochem. 34 (2001) 107

[18] J. Suttnar, L. Mosova, J.E. Dyr, J. Chromatogr. B 751 (2001) 193.

[19] G. Charlot, Les Réactions Chimiques en Solution Aqueuse et Caractérisation des Ions, Masson, Paris, 1983.

[20] F. Ricci, G. Palleschi, Biosens. Bioelectr. 21 (2005) 389.

[21] F. Ricci, D. Moscone, C.S. Tuta, G. Palleschi, A. Amine, A. Poscia, F. Valgimigli, D. Messeri, Biosens. Bioelectr. 20 (2005) 1993. 